\title{
Cigarrinhas-das-pastagens em Santa Catarina: avaliação do complexo de espécies e da incidência natural de fungos entomopatogênicos
}

\author{
Leandro do Prado Ribeiro e Angélica Ribolli Cazarotto²
}

\begin{abstract}
Resumo - Levantamentos sistemáticos foram realizados de modo a determinar a proporção de espécies no complexo de cigarrinhas-das-pastagens incidentes em pastagens estabelecidas em diferentes municípios de Santa Catarina e avaliar a incidência natural de fungos entomopatogênicos associados aos espécimens coletados. Para isso, cigarrinhas adultas foram coletadas em pastagens de grama-bermuda, Cynodon dactylon cv. Tifton 85, sem histórico da aplicação de micoinseticidas, em 12 municípios do estado usando rede de varredura. Em laboratório, a proporção de espécies de cigarrinhas foi determinada e a mortalidade natural observada durante dez dias, sendo os exemplares mortos desinfectados e mantidos em placas de Elisa para avaliação da incidência de fungos, com posterior isolamento dos mesmos. Com base nas amostras obtidas, foram identificadas sete espécies de cigarrinhas (Notozulia entreriana, Deois schach, Deois knoblauchii, Mahanarva integra, Deois flavopicta, Deois flexuosa e Deois incompleta), sendo N. entreriana e D. schach classificadas como superfrequentes, constantes, superdominantes e superabundantes de acordo com a análise faunística empregada. Além disso, foram encontradas 15 espécies de fungos em associação às cigarrinhas (Alternaria sp., Aspergillus sp., Aspergillus niger, Bipolaris sp., Cladosporium sp., Curvularia sp., Metarhizium sp., Mucor sp., Nigrospora sp., Penicillium sp., Pestalotia sp., Pithomiices sp. e Monilia sp.), sendo a maioria delas reconhecidas como patógenos oportunistas, saprofitos ou fungos endofíticos. A incidência natural do fungo entomopatogênico Metarhizium spp. foi baixa em todas as áreas amostradas ( $0,7 \%$ do total de cigarrinhas coletadas).
\end{abstract}

Termos para indexação: Cercopidae; amostragem populacional; Notozulia entreriana; Deois schach; controle microbiano.

\section{Spittlebugs in Santa Catarina: assessment of species complex and natural incidence of entomopathogenic fungi}

\begin{abstract}
Systematic surveys were conducted to determine the proportion of species of spittlebugs and assess the natural incidence of entomopathogenic fungi species on populations collected in pastures located in different municipalities of the state of Santa Catarina, Brazil. For such, adult specimens were collected in pastures of bermudagrass, Cynodon dactylon cv. Tifton 85, without application of mycoinsecticides from 12 municipalities using a sweeping net device. In the laboratory conditions, the proportion of spittlebugs species and their natural mortality was observed for 10 days. Their cadavers were stored and disinfected, being kept on Elisa plates to assess the incidence of fungi species and their subsequent isolation. Based on obtained samples, seven spittlebug species were identified (Notozulia entreriana, Deois schach, Deois knoblauchii, Mahanarva integra, Deois flavopicta, Deois flexuosa, Deois incompleta), being $N$. entreriana and D. schach classified as superfrequent, constant, super-dominant and super-abundant according to the faunistic analysis employed. Moreover, 15 fungi species were found (Alternaria sp., Aspergillus sp., A. niger, Bipolaris sp., Cladosporium sp., Curvularia sp., Metarhizium sp., Mucor sp., Nigrospora sp., Penicillium sp., Pestalotia sp., Pithomiices sp., Monilia sp.) in association with spittlebugs. Most are recognized as opportunistic pathogens or plant endophytes. Natural incidence of entomopathogenic fungus Metarhizium spp. was low in all sampled areas ( $0.7 \%$ of the total of collected spittlebug).
\end{abstract}

Index terms: Cercopidae; populational sampling; Notozulia entreriana; Deois schach; microbial control.

\section{Introdução}

As cigarrinhas-das-pastagens (Hemiptera: Auchenorrhyncha: Cercopidae) constituem um complexo de espécies que se configuram na principal praga de gramíneas (Poaceae) forrageiras em toda a América Latina (ALVARENGA et al., 2017; PALADINI et al., 2018). Os danos causados pelas cigarrinhas são verificados tanto na redução do volume de matéria seca produzida (dano quantitativo), que limitam consideravelmente a capacidade de suporte das pastagens (AUAD et al., 2007), quanto na qualidade nutricional dos tecidos da planta (dano qualitativo) (VALÉRIO \& NAKANO, 1987, 1988; CONGIO et al., 2012). Além disso, o ataque intenso de pragas é uma das causas da degradação de áreas de pastagens, problema crônico da pecuária brasileira que ocasiona prejuízos para toda a sociedade (VALÉRIO et al., 2000; PERON \& EVANGELISTA, 2004).•

${ }^{2}$ Engenheira-agrônoma, Universidade Federal da Fronteira Sul (UFFS), Chapecó, Santa Catarina. E-mail: angelica.ribollicazarotto@gmail.com. 
A distribuição e a proporção das espécies de cigarrinhas-das-pastagens são bastante variáveis de acordo com as condições climáticas, espécies hospedeiras (nível de resistência) e histórico de uso e manejo da área (RIBEIRO \& CASTILHOS, 2018). No Brasil, a espécie de cercopídeo Deois flavopicta Stal é a que ocorre com maior frequência, especialmente nas regiões Sudeste e CentroOeste (FONTES et al.,1995; VALÉRIO et al., 2000). No Oeste Catarinense, Bertollo et al. (2007) verificaram que Zulia entreriana (Berg) (atualmente Notozulia entreriana) é a espécie predominantemente encontrada em pastagens formadas por gramíneas perenes de verão, embora os autores tenham verificado diferenças na ocorrência e na proporção das espécies de acordo com a gramínea hospedeira. Em grama-missioneira-gigante (Axonopus catharinensis Valls), $D$. flavopicta e Deois schach (F.) foram as espécies mais abundantemente encontradas em Chapecó, SC (CHIARADIA et al., 2013).

No Brasil, o controle químico do complexo de cigarrinhas-das-pastagens tem sido a principal estratégia de manejo adotada pelos agricultores (RIBEIRO \& CASTILHOS, 2018). Em contrapartida, o controle microbiano das cigarrinhasdas-pastagens com o fungo entomopatogênico Metarhizium anisopliae (Metchnikoff) Sorokin (Ascomycota: Clavicipitaceae) vem sendo cada vez mais utilizado em virtude da crescente disponibilidade de formulações comerciais registradas (LOVATTO \& RIBEIRO, 2017; MAINA et al., 2018). Além de M. anisopliae, outras espécies de fungos podem contribuir para o controle natural das cigarrinhas-das-pastagens. Leite et al. (2002) encontraram Furia sp. (Entomophthorales) em níveis epizoóticos atacando $D$. schach em pastagem de Brachiaria, provocando até $80 \%$ de infecção, com consequente queda na população do inseto. Nesse mesmo estudo, os autores também constataram Batkoa sp. (Entomophthorales) atacando Mahanarva fimbrioata (Stal) em pastagem de capim-elefante $\mathrm{cv}$. Napier. $\mathrm{Na}$
Argentina, Foieri et al. (2018) relataram a ocorrência natural do fungo do gênero Pandora (Entomophthorales) sobre populações de cercopídeos-praga.

Diante do exposto, este estudo teve como objetivos avaliar, por meio de índices faunísticos, a frequência, constância, dominância e abundância das espécies no complexo de cigarrinhas-daspastagens incidentes em pastagens perenes de verão de propriedades leiteiras de diferentes regiões de Santa Catarina, assim como a incidência natural de espécies de fungos entomopatogênicos associadas a essas populações. Além de contribuir para o estabelecimento de uma coleção de isolados de fungos entomopatogênicos, esse conjunto de informações subsidiará futuros estudos que busquem potencializar o controle microbiano aplicado desse importante complexo de espécies-praga e o desenvolvimento de programas de melhoramento genético visando à obtenção de genótipos resistentes para as espécies majoritariamente encontradas nas principais regiões produtoras.

\section{Material e métodos}

As coletas, visando avaliar a proporção de espécies no complexo de cigarrinhas-das-pastagens e a incidência natural de fungos entomopatogênicos associados, foram realizadas em pastagens estabelecidas em propriedades produtoras de leite de 12 municípios (Alto da Bela Vista, Arvoredo, Bom Retiro, Brunópolis, Campo Erê, Canoinhas, lomerê, Lindóia do Sul, Ouro Verde, Palmitos, Santa Rosa do Sul e São Miguel do Oeste) de diferentes regiões de Santa Catarina (Figura 1). Os levantamentos foram realizados entre os meses de janeiro e março de 2016, em áreas de pastagens constituídas por Cynodon dactylon cv. Tifton 85 [uma das forrageiras mais cultivadas no Estado (JOCHIMS et al., 2017)] sem histórico de uso de micoinseticidas. A determinação dos pontos de amostragem levou em consideração a distância entre eles ( 100km) e a representatividade da atividade leiteira em cada região. Cada ponto amostrado foi georreferenciado utilizando-se

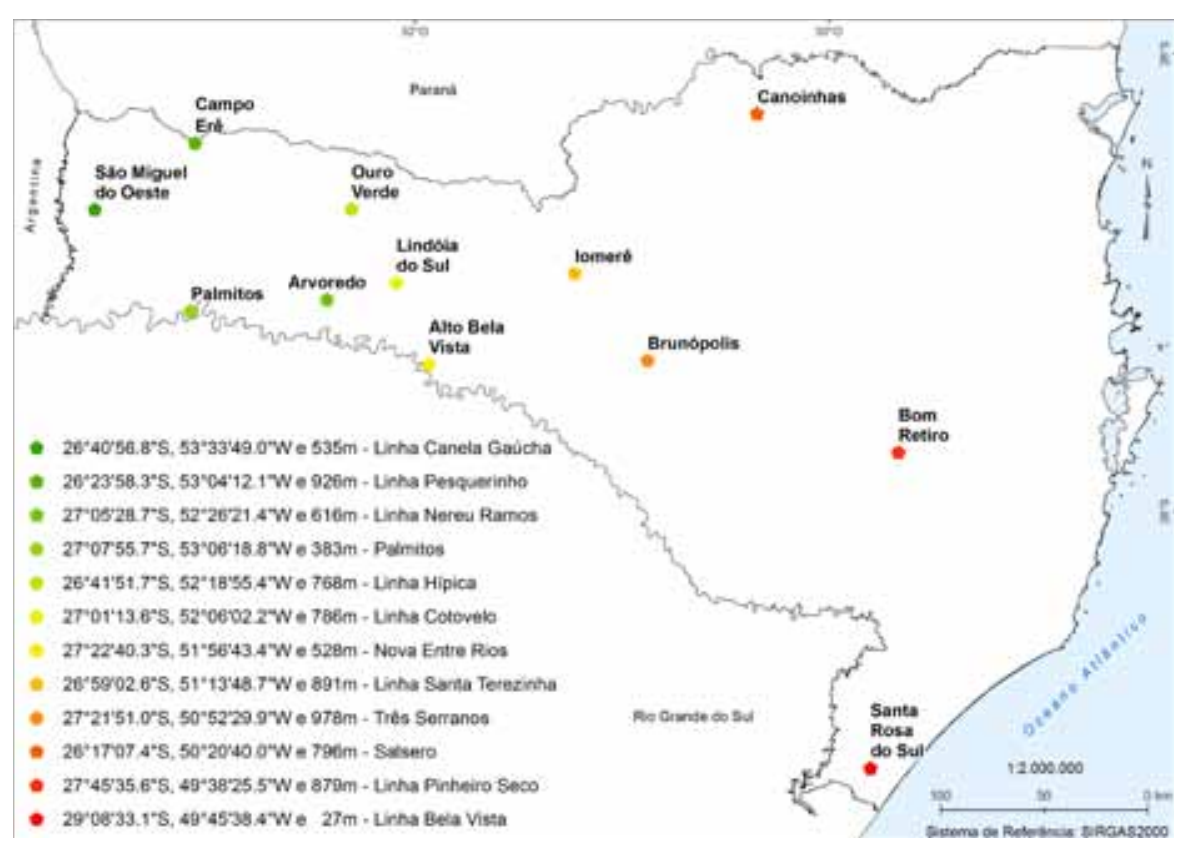

Figura 1. Municípios de Santa Catarina onde foram coletadas amostras de cigarrinhas-daspastagens visando à avaliação da proporção de espécies e ocorrência natural de fungos entomopatogênicos

Figure 1. Municipalities of Santa Catarina, Brazil where samples of spittlebugs were collected in order to evaluate the proportion of species as well as the natural occurrence of entomopathogenic fungi 
um GPS de navegação Garmin Etrex (Garmin Brasil Comércio de Tecnologias Ltda., Barueri, SP).

Para coleta das cigarrinhas adultas foi utilizada rede de varredura com $40 \mathrm{~cm}$ de diâmetro. Cada amostra foi composta por indivíduos coletados por meio de redadas em movimentos pendulares em pontos aleatoriamente distribuídos de cada área com esforço amostral de 30 minutos. Para transporte ao laboratório, os insetos coletados vivos foram dispostos em caixas teladas $(30 \times 30 \times 30 \mathrm{~cm})$ de modo a permitir a livre circulação do ar e contendo partes da forrageira da própria área amostrada para servir de sítio de refúgio e alimentação durante o transporte. As coletas e transportes das cigarrinhas-das-pastagens foram realizadas com prévia autorização emitida pelo Sistema de Autorização e Informação em Biodiversidade (ICMBio/SISBIO) do Instituto Brasileiro do Meio Ambiente e Recursos Naturais Renováveis do Ministério do Meio Ambiente (IBAMA/MMA), sob número 51520-1.

\section{Avaliação da proporção de espécies e incidência de fungos associados}

Em laboratório, as cigarrinhas foram separadas por espécies (morfotipos) e locais amostrados, sendo que vouchers specimens foram encaminhados à Profa. Dra. Andressa Paladini (UFSM, Santa Maria, RS) visando à confirmação específica. As cigarrinhas coletadas (vivas) foram mantidas em gaiolas confeccionadas com tecido fino do tipo voile, dispostas sobre plantas de Brachiaria brizantha (Poaceae) cultivadas em vasos com capacidade de 15 litros. Diariamente, por um período de dez dias, foi observada a ocorrência de cigarrinhas mortas, as quais foram desinfectadas em solução de hipoclorito de sódio a $0,5 \%\left(v v^{-1}\right)$ por 30 segundos seguido de três lavagens em água destilada estéril. Feito isso, essas foram transferidas, imediatamente, para placas de Elisa (24 células) visando à confirmação da mor- talidade pela constatação da extrusão do patógeno, distinguido pelo crescimento micelial característico sobre o tegumento do inseto após sete dias de incubação.

Em câmara asséptica, o isolamento dos fungos foi realizado através do método de plaqueamento em meio de cultura BDA (batata, dextrose e ágar). Para isso, o meio preparado foi autoclavado e adicionado às placas de Petri $(9 \mathrm{~cm}$ de diâmetro) previamente esterilizadas. 0 isolamento dos fungos observados nos exemplares mortos foi realizado por meio da raspagem da superfície das cigarrinhas contaminadas com uma pinça esterilizada, introduzindo, posteriormente, o micélio do fungo no meio de cultura. As placas foram incubadas em câmaras tipo BOD, com temperatura de $25 \pm 2^{\circ} \mathrm{C}$, umidade relativa de $60 \pm 10 \%$ e fotoperíodo de 12 horas, por sete dias. Nas placas em que houve o crescimento de mais de um tipo de fungo foi realizada a repicagem, separando-os em placas contendo meio de cultura BDA e mantendo-os em câmara BOD sob as mesmas condições mencionadas anteriormente. A identificação das espécies de fungos foi realizada utilizando-se chaves de classificação. Alguns isolados selecionados foram preservados em tubos criogênicos com glicerol 10\%, mantidos em ultrafreezer $\left(-80^{\circ} \mathrm{C}\right)$ e depositados na coleção de fungos entomopatogênicos da Epagri/Cepaf.

\section{Análise dos dados obtidos}

Inicialmente, os dados obtidos foram submetidos a análises descritivas. Por sua vez, os índices de frequência, abundância, dominância e constância das espécies de cigarrinhas-das-pastagens foram obtidos por meio de análise faunística utilizando o software ANAFAU (MORAES et al., 2003). Na classificação do ANAFAU é adicionada a classe extrema (super), referente aos valores discrepantes de número de insetos, discriminados através da análise de resíduos. Esses valores não são considerados no cálculo dos parâmetros de dominância, frequência e abundância. Além disso, a diversidade de cigarrinhas-das-pastagens foi calculada pelo índice de Shannon-Wiener $\left(\mathrm{H}^{\prime}\right)$. Por sua vez, o índice de riqueza de espécies adotado foi de Margalef $(\alpha)$ e, para estimar a uniformidade em termos de abundância de indivíduos entre as espécies amostradas, foi calculado o índice de equitabilidade ( $E^{\prime}$ ) (SILVEIRA NETO et al., 1976).

\section{Resultados e discussão}

Com base nos espécimens adultos amostrados ( $n=1564)$ em pastagens de grama-bermuda cultivadas em diferentes municípios de Santa Catarina, foram identificadas sete espécies de cercopídeos (Hemiptera: Auchenorrhyncha) compondo o complexo de cigarrinhas-das-pastagens (Figura 1, Tabela 1). Embora tenham sido verificadas diferenças na proporção das espécies de cigarrinhas-das-pastagens entre os locais amostrados (Tabela 1 ), $N$. entreriana foi a espécie mais abundante, correspondendo por $74,04 \%$ dos indivíduos coletados (Figuras 1 e 2), seguido por Deois schach $(19,50 \%$ dos indivíduos coletados). Essas duas espécies foram as únicas classificadas como superfrequentes, constantes, superdominantes e superabundantes, de acordo com a análise faunística empregada (Tabela 2).

$O$ índice de Shannon-Weaner estimado $(1,0323)$ foi baixo, o que denota a ocorrência de fatores limitantes e de competição interespecífica intensa nos locais amostrados (SILVEIRA NETO et al., 1976). Nesse sentido, as espécies mais comuns aumentam suas populações e as espécies raras apresentam baixo nível populacional (HUSCH et al., 2012). A riqueza das espécies, medida pelo índice de Margalef $(0,8667)$, confirma haver a predominância de espécies, ou seja, a ocorrência de poucas espécies com populações de muitos indivíduos, com destaque para $N$. entreriana, que correspondeu a mais de $70 \%$ dos exemplares coletados. $O$ índice de equitabilidade ou uniformidade estimada foi de 0,6414 , sendo que quanto mais próximo 
Tabela 1. Locais amostrados e proporção de espécies de cigarrinhas-da-pastagens (Hemiptera: Auchenorrhyncha: Cercopidae) coletadas em pastagens de Cynodon dactylon cv. Tifton 85 em 12 municípios de Santa Catarina (janeiro a março de 2016)

Table 1. Sampling sites and proportion of spittlebugs species (Hemiptera: Auchenorrhyncha: Cercopidae) collected on pastures of Cynodon dactylon Cv. Tifton 85 from 12 municipalities of Santa Catarina, Brazil (January to March 2016)

\begin{tabular}{|c|c|c|c|c|c|c|c|c|}
\hline \multirow[b]{2}{*}{ Locais amostrados } & \multirow{2}{*}{$\begin{array}{l}\text { Número de } \\
\text { insetos } \\
\text { coletados }\end{array}$} & \multicolumn{7}{|c|}{ Proporção de espécies coletadas (\%) } \\
\hline & & $\begin{array}{l}\text { Notozulia } \\
\text { entreriana }\end{array}$ & $\begin{array}{l}\text { Deois } \\
\text { schach }\end{array}$ & $\begin{array}{c}\text { Deois } \\
\text { knoblauchii }\end{array}$ & $\begin{array}{l}\text { Mahanarva } \\
\text { integra }\end{array}$ & $\begin{array}{c}\text { Deois } \\
\text { flavopicta }\end{array}$ & $\begin{array}{l}\text { Deois } \\
\text { flexuosa }\end{array}$ & $\begin{array}{c}\text { Deois } \\
\text { incompleta }\end{array}$ \\
\hline Alto da Boa Vista & 127 & 88,98 & 8,66 & 2,36 & 0,00 & 0,00 & 0,00 & 0,00 \\
\hline Arvoredo & 252 & 81,35 & 0,79 & 15,48 & 0,40 & 1,98 & 0,00 & 0,00 \\
\hline Bom Retiro & 83 & 0,00 & 98,80 & 1,20 & 0,00 & 0,00 & 0,00 & 0,00 \\
\hline Brunópolis & 73 & 86,30 & 13,70 & 0,00 & 0,00 & 0,00 & 0,00 & 0,00 \\
\hline Canoinhas & 3 & 0,00 & 100,00 & 0,00 & 0,00 & 0,00 & 0,00 & 0,00 \\
\hline Campo Erê & 238 & 13,44 & 76,05 & 6,73 & 0,00 & 3,78 & 0,00 & 0,00 \\
\hline lomerê & 375 & 97,34 & 0,53 & 0,00 & 2,13 & 0,00 & 0,00 & 0,00 \\
\hline Lindóia do Sul & 99 & 96,97 & 1,01 & 0,00 & 1,01 & 1,01 & 0,00 & 0,00 \\
\hline Ouro Verde & 282 & 92,55 & 3,90 & 1,42 & 2,13 & 0,00 & 0,00 & 0,00 \\
\hline Palmitos* & 0 & -- & -- & -- & -- & -- & -- & -- \\
\hline Santa Rosa do Sul & 20 & 60,00 & 10,00 & 5,00 & 0,00 & 0,00 & 20,00 & 5,00 \\
\hline $\begin{array}{l}\text { São Miguel do } \\
\text { Oeste }\end{array}$ & 12 & 91,67 & 0,00 & 8,33 & 0,00 & 0,00 & 0,00 & 0,00 \\
\hline Total & 1564 & 74,04 & 19,50 & 4,16 & 1,02 & 0,96 & 0,26 & 0,06 \\
\hline
\end{tabular}

* Na data de amostragem realizada, não foram encontradas cigarrinhas-das-pastagens adultas na área amostrada.

Tabela 2. Análise faunística do complexo de cigarrinhas-da-pastagens (Hemiptera: Auchenorrhyncha: Cercopidae) em pastagens de Cynodon dactylon cv. Tifton 85 de 12 municípios de Santa Catarina (janeiro a março de 2016)

Table 2. Faunistic analysis of spittlebugs complex (Hemiptera: Auchenorrhyncha: Cercopidae) in pastures of Cynodon dactylon cv. Tifton 85 from 12 municipalities of Santa Catarina, Brazil (January to March 2016)

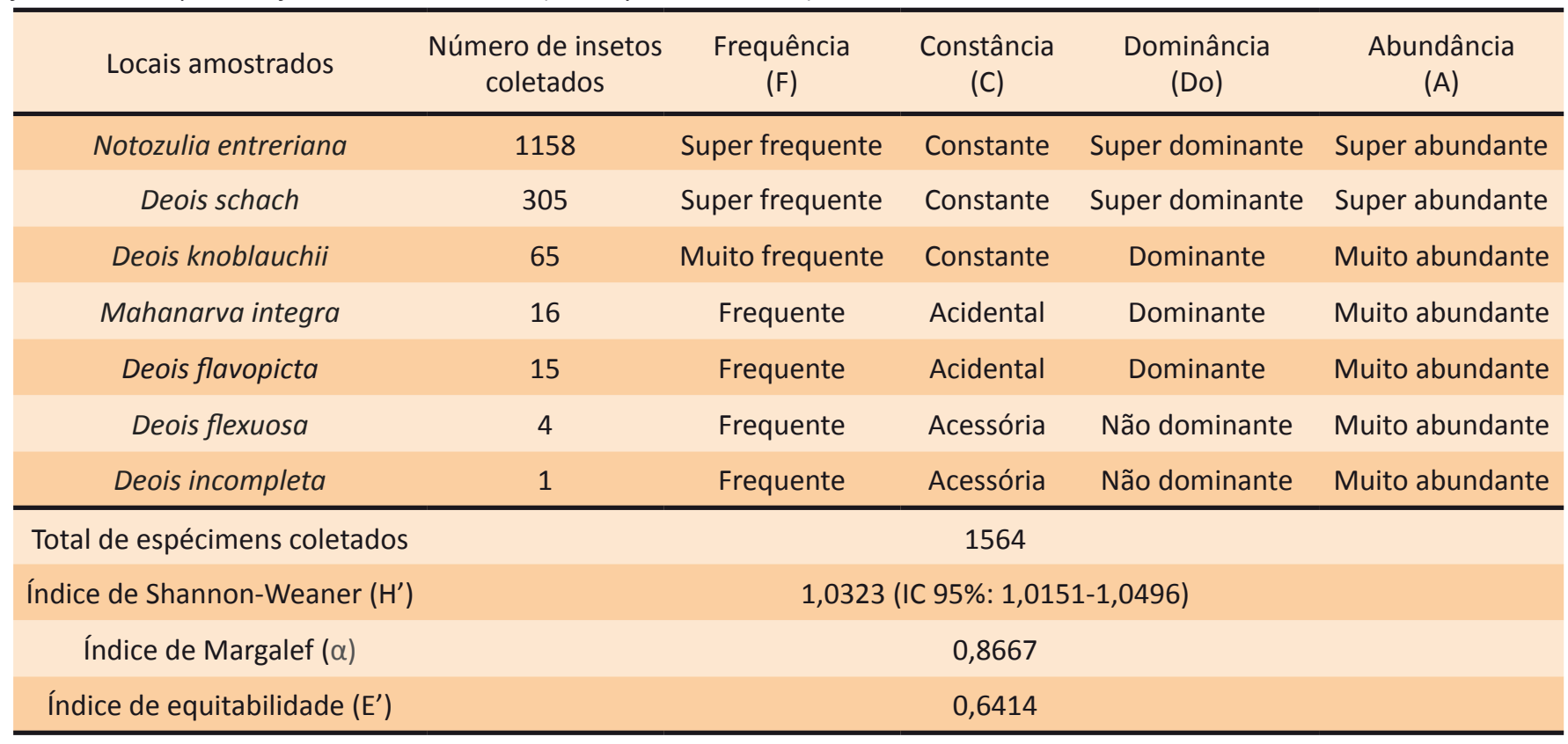


a zero maior o indicativo da predominância de espécies (HUSCH et al., 2012).

Os resultados obtidos nos diferentes locais amostrados indicam a predominância das espécies $N$. entreriana e $D$. schach em pastagens de $C$. dactylon cv. Tifton 85, diferindo de levantamentos prévios realizados em Santa Catarina (BERTOLLO et al., 2007; CHIARADIA et al., 2013). Apesar da distribuição e da proporção das espécies de cigarrinhasdas-pastagens ser bastante variável de acordo com as condições climáticas, as espécies forrageiras hospedeiras e o histórico de uso e manejo da área (RIBEIRO \& CASTILHOS, 2018), os resultados obtidos indicam que essas duas espécies deverão ser, preferencialmente, o alvo de futuros estudos visando o desenvolvimento de genótipos resistentes ou o delineamento de estratégias de manejo integrado.

Entre os espécimens adultos coletados, 56,65\% apresentaram mortalidade dentro do período observado (dez dias), e ocorrência de extrusão de estruturas de patógenos associados (Tabela 3). Assim, foram identificadas 15 espécies de fungos em associação às cigarrinhas (Alternaria sp., Aspergillus sp., A. niger, Bipolaris sp., Cladosporium sp., Curvularia sp., Fusarium sp., Metarhizium sp., Mucor sp., Nigrospora sp., Papulospora sp., Penicillium sp., Pestalotia sp., Pithomiices sp. e Monilia sp.) (Tabela 3), sendo a maioria deles reconhecidos como patógenos oportunistas ou fungos endofíticos (HUANG \& JIANG, 2015; KAUR et al., 2015). A incidência natural do fungo entomopatogênico Metarhizium spp. foi baixa em todas as áreas amostradas, correspondendo a $0,7 \%$ do total de cigarrinhas coletadas, ou seja, apenas $1,24 \%$ dos insetos infectados das amostras obtidas.

Bipolaris sp. (29,89\%), Fusarium sp. (22,35\%), Nigrospora sp. (9,93\%) e Curvularia sp. $(9,14)$ foram as espécies de fungos mais abundantemente associadas às populações de cigarrinhas-daspastagens (Tabela 3). Embora muitas espécies desses gêneros constituam patógenos de $C$. dactylon e esporos des-

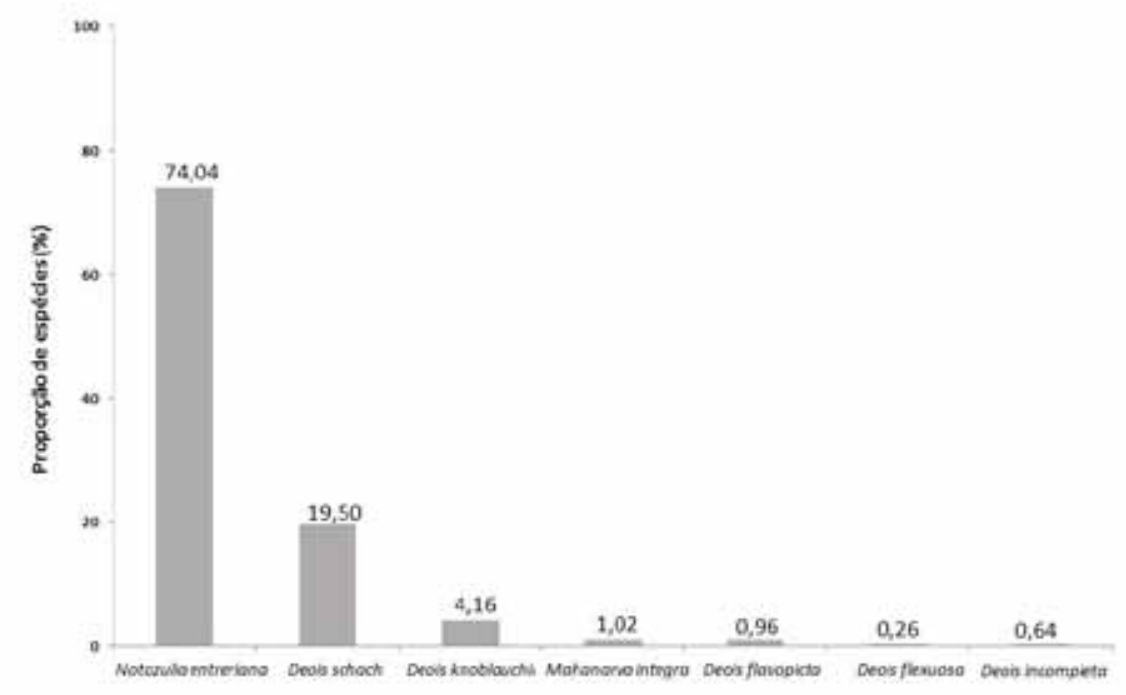

Figura 2. Proporção de espécies de cigarrinhas-da-pastagens (Hemiptera: Auchenorrhyncha: Cercopidae) em pastagens de Cynodon dactylon cv. Tifton 85 em 12 municípios de Santa Catarina (janeiro a março de 2016)

Figure 2. Proportion of spittlebugs species (Hemiptera: Auchenorrhyncha: Cercopidae) on pastures of Cynodon dactylon cv. Tifton 85 from 12 municipalities of Santa Catarina, Brazil (January to March 2016)

Tabela 3. Espécies de fungos associados ao complexo de cigarrinhas-da-pastagens (Hemiptera: Auchenorrhyncha: Cercopidae) coletadas em pastagens de Cynodon dactylon cv. Tifton 85 de 12 municípios de Santa Catarina $(n=1564)$

Table 3. Fungal species associated to spittlebugs complex (Hemiptera: Auchenorrhyncha: Cercopidae) collected on pastures of Cynodon dactylon cv. Tifton 85 from 12 municipalities of Santa Catarina, Brazil $(n=1564)$

\begin{tabular}{|c|c|c|}
\hline Espécie identificada & $\begin{array}{c}\text { Número de insetos } \\
\text { infectados }\end{array}$ & $\begin{array}{c}\% \text { de insetos } \\
\text { infectados }\end{array}$ \\
\hline Alternaria sp. & 59 & 6,66 \\
\hline Aspergillus sp. & 8 & 0,90 \\
\hline Aspergillus niger & 7 & 0,79 \\
\hline Bipolaris sp. & 256 & 28,89 \\
\hline Cladosporium sp. & 23 & 2,60 \\
\hline Curvularia sp. & 81 & 9,14 \\
\hline Fusarium sp. & 198 & 22,35 \\
\hline Metarhizium sp. & 11 & 1,24 \\
\hline Mucor sp. & 44 & 4,97 \\
\hline Nigrospora sp. & 88 & 9,93 \\
\hline Papulospora sp. & 3 & 0,34 \\
\hline Penicillium sp. & 14 & 1,58 \\
\hline Pestalotia sp. & 8 & 0,90 \\
\hline Pithomiices sp. & 2 & 0,23 \\
\hline Monilia sp. & 1 & 0,11 \\
\hline Não identificadas & 83 & 9,37 \\
\hline Total & \multicolumn{2}{|c|}{886} \\
\hline $\begin{array}{c}\text { Proporção de insetos } \\
\text { infectados }\end{array}$ & \multicolumn{2}{|c|}{$56,65 \%$} \\
\hline
\end{tabular}




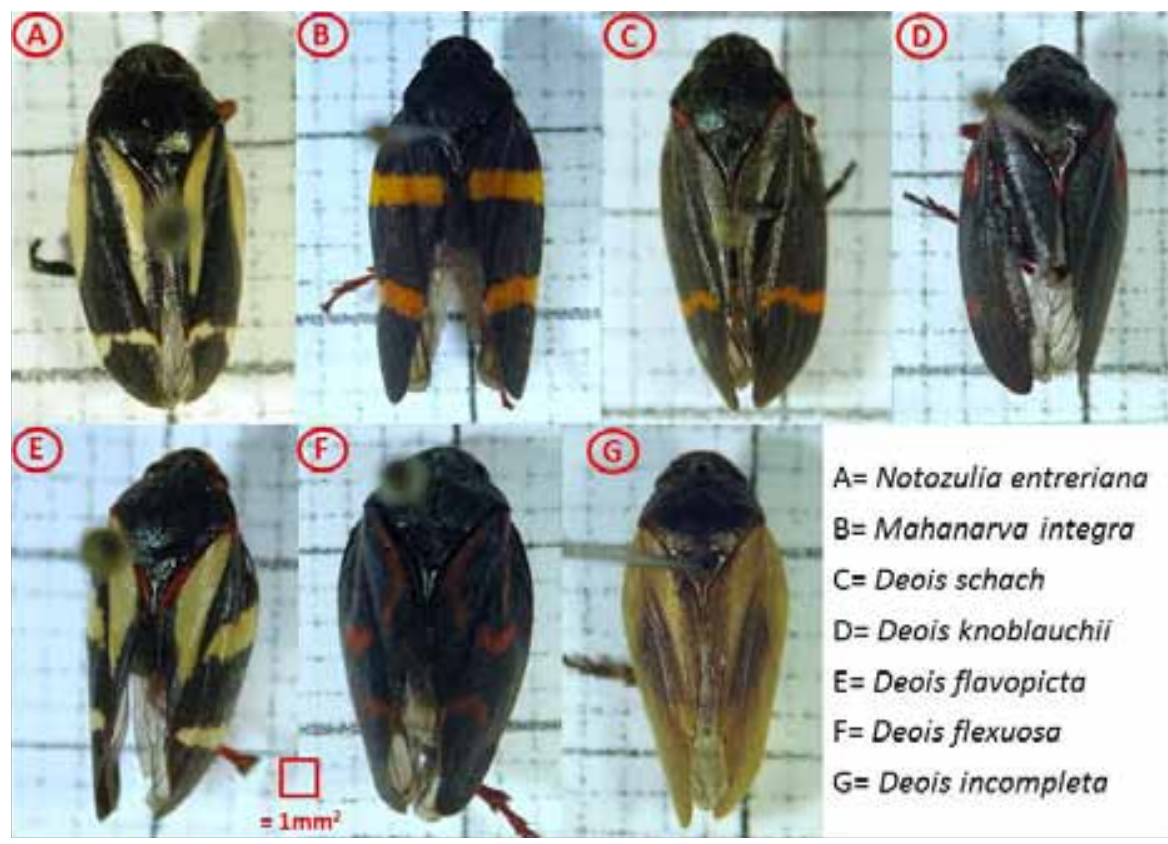

Figura 3. Espécies de cigarrinhas-da-pastagens (Hemiptera: Auchenorrhyncha: Cercopidae) incidentes em pastagens de Cynodon dactylon cv. Tifton 85 em 12 municípios de Santa Catarina

Figure 3. Species of spittlebugs (Hemiptera: Auchenorrhyncha: Cercopidae) on pastures of Cynodon dactylon cv. Tifton 85 from 12 municipalities of Santa Catarina, Brazil

sas espécies possam estar associados ao sistema digestório das cigarrinhas, estudos têm demonstrado o efeito de espécies desses gêneros ou mesmo de metabólitos secundários por eles produzidos sobre insetos. Por exemplo, Pečiulytè et al. (2010) verificaram a ação de isolados de Lecanicillium psalliotae (syn. Verticillium psalliotae) e Fusarium solani sobre Bupalus piniaria L. (Lepidoptera: Geometridae). Isolado de Fusarium pallidoroseum, obtidos de cadáveres de Lymantria dispar (L.) (Lepidoptera: Erebidae), causaram promissores níveis de mortalidade de lagartas de L. dispar (MUNSHI et al., 2008). A toxina beauvericin produzida por isolado de Fusarium semitectum e Fusarium moniliforme var. subglutinans apresenta toxicidade para o besouro Leptinotarsa decemlineata (Say) (Coleoptera: Chrysomelidae) (GUPTA et al., 1991). Thakur et al. (2014) verificaram que os fungos endofíticos Nigrospora oryzae e Cladosporium uredinicola apresentam efeito citotóxico sobre hemócitos de Spodoptera litura (F.) (Lepidoptera: Noctuidae), alterando seu sistema de defesa. Poste- riormente, o mesmo efeito também foi demonstrado a partir do fungo endofítico Alternaria alternata (KAUR et al., 2015).

Recentemente, Campagnani et al. (2017) detectaram a ocorrência de cinco isolados de fungos associados a Mahanarva spectabilis (Distant) (Hemiptera: Cercopidae) em áreas de sistemas silvipastoris (SSP) tropicais: Penicillium sp. (isolados UFMG 11440 e UFMG 11441), Mucor sp. (UFMG 11441), Fusarium sp. (isolado UFMG 11443) e Metarhizium sp. (isolado 11444). Em ensaios de virulência, os autores verificaram que os isolados UFMG 11443 e 11444 causaram mais de $90 \%$ de mortalidade de ovos e de ninfas expostas, enquanto que os demais isolados (UFMG 11440, 11441 e 11442) foram igualmente ou mais eficientes que a cepa comercial de M. anisopliae (controle positivo), com mais de $50 \%$ de ovos inviáveis ou mortalidade de ninfas. Diante desse potencial, estudos de virulência deverão ser conduzidos futuramente com os isolados obtidos no levantamento realizado de modo a avaliar o potencial desses isola- dos selvagens no controle biológico das espécies de cigarrinhas-das-pastagens mais abundantes em Santa Catarina. Todavia, estudos em condições de campo também deverão ser conduzidos de modo a avaliar a bioatividade e o comportamento desses isolados em condições naturais, subsidiando a avaliação do potencial uso desses isolados na formulação de micoinseticidas.

\section{Conclusões}

Com base nos levantamentos realizados nas condições pré-estabelecidas, conclui-se que:

- Em pastagens de grama-bermuda (C. dactylon cv. Tifton 85), sete espécies ( $N$. entreriana, D. schach, D. knoblauchii, M. integra, D. flavopicta, D. flexuosa e $D$. incompleta) compõem o complexo de cigarrinhas-das-pastagens incidentes em Santa Catarina;

- N. entreriana e D. schach são espécies superfrequentes, constantes, superdominantes e superabundantes em pastagens de grama-bermuda nas regiões amostradas de Santa Catarina;

- A incidência natural do fungo entomopatogênico Metarhizium spp. é baixa em todas as áreas amostradas $(0,7 \%$ do total de cigarrinhas coletadas).

\section{Agradecimentos}

À Zelinda Meneguzzi, Carmen Cella dos Santos e Neusa Terezinha Maciel (Epagri/Cepaf) pela colaboração técnica na execução do estudo. Aos técnicos de campo da Epagri/Cepaf e dos escritórios municipais da Epagri de diferentes municípios pelo auxílio na coleta de cigarrinhas-das-pastagens nas propriedades amostradas. Os autores também agradecem à prof. Dra. Andressa Paladini (UFSM) pela identificação das espécies de cigarrinhas, ao Dr. Wiliam da Silva Ricce (Epagri/Ciram) pelo auxílio na elaboração do mapa dos locais amostrados e a Regina Célia Botequio de Moraes (Departamento de Entomologia, ESALQ/USP) pelo auxílio na análise faunística. Por fim, os autores agradecem 
ao CNPq (Processo 445518/2014-6) pelo suporte financeiro.

\section{Referências}

ALVARENGA, R.; AUAD, A. M.; MORAES, J. C.; SILVA, S. E. B.; RODRIGUES, B. S.; SILVA, G. B. Spittlebugs (Hemiptera: Cercopidae) and their host plants: a strategy for pasture diversification. Applied Entomology and Zoology, [S.I.], v. 52, n. 4, p. 653-660, 2017.

AUAD, A. M.; SIMÕES, A. D.; PEREIRA, A. V.; BRAGA, A. L. F; SOBRINHO, F. S.; LÉDO, F. J. S.; PAULA-MORAES, S. V.; OLIVEIRA, S. A.; FERREIRA, R. B. Seleção de genótipos de capim-elefante quanto à resistência à cigarrinha-das-pastagens. Pesquisa Agropecuária Brasileira, Brasília, DF, v. 42, n. 8, p. 1077-1081, 2007.

BERTOLLO, E. C.; MILANEZ, J. M.; CHIARADIA, L. A. Ocorrência e flutuação populacional de cigarrinhas-das-pastagens em diferentes espécies de gramíneas. Agropecuária Catarinense, Florianópolis, v. 20, n. 1, p. 8286, 2007.

CAMPAGNANI, M. O.; CAMPOS, W. G.; AMORIM, S. S.; ROSA, L. H.; AUAD, A. M.; CANGUSSÚ, M. A.; MAURÍCIO, R. M. Prospection and fungal virulence associated with Mahanarva spectabilis (Hemiptera: Cercopidae) in an Amazon silvopastoral system. Florida Entomologist, Florida, v. 100 , n. 2, p. 426-432, 2017.

CHIARADIA, L. A.; MIRANDA, M.; FEDATTO, V. J. Flutuação populacional de cigarrinhasdas-pastagens na grama-missioneira-gigante. Agropecuária Catarinense, Florianópolis, v. 26, p. 70-75, 2013.

CONGIO, G. F. S.; CORSI, M.; ALMEIDA, P. C.; GOULART, R. C. D.; PASSOS, L. P.; KÖPP, M. M. Rebrota do capim-marandu submetido ao ataque de cigarrinhas. Arquivos do Instituto Biológico, São Paulo, v. 79, n. 3, p. 389-396, 2012.

FOIERI, A.; PEDRINI, N.; TOLEDO, A. Natural occurrence of the entomopathogenic genus Pandora on spittlebug pests of crops and pastures in Argentina. Journal of Applied Entomology, [S.I.], v. 142, n. 3, p. 363-370, 2018.

FONTES, E. M. G.; PIRES, C. S. S.; SUJII, E. R. Mixed risk-spreading strategies and the population dynamics of a Brazilian pasture pest, Deois flavopicta (Homoptera: Cercopidae). Journal of Economic Entomology, Lanham, v. 88, n. 5, p. 1256-1262, 1995.

GUPTA, S.; KRASNOFF, S. B.; UNDERWOOD, N. L.; RENWICK, J. A. A.; ROBERTS, D. W. Isolation of beauvericin as an insect toxin from Fusarium semitectum and Fusarium moniliforme var. subglutinans. Mycopathologia, [S.I.], v. 115, n. 3, p. 185-189, 1991.

HUANG, J.; JIANG, Z. Phylogenetic analysis of endophytes from bitter Melon (Momordica charantia) in Guangdong Province. Agricultural Sciences, [S.I.], v. 6, p. 609-621, 2015.

HUSCH, P. E.; MILLÉO, J.; SEDORKO, D.; AYUB, R. A.; NUNES, D. S. Caracterização da fauna de moscas-das-frutas (Diptera: Tephritidae) na região de Ponta Grossa, Paraná, Brasil. Ciência Rural, Santa Maria, v. 42, n. 10, p. 1833-1839, 2012.

JOCHIMS, F.; SILVA, A. W. L.; PORTES, V. M. Espécies forrageiras mais utilizadas em pastagens na região Oeste de Santa Catarina. Agropecuária Catarinense, Florianópolis, v. 30, n. 3, p. 15-18, 2017.

KAUR, H. P.; SINGH, B.; THAKUR, A.; KAUR, A.; KAUR, S. Studies on immunomodulatory effect of endophytic fungus Alternaria alternata on Spodoptera litura. Journal of AsiaPacific Entomology, [S.I.], v. 18, n. 1, p. 6775, 2015.

LEITE, L. G.; ALVES, S. B.; TAKADA, H. M.; BATISTA FILHO, A.; ROBERTS, D. W. Ocurrence of Batkoa sp. and Furia sp. fungi in pasture spittlebug populations in eastern São Paulo State, Brazil. Arquivos do Instituto Biológico, São Paulo, v. 69, n. 3, p. 63-68, 2002.

LOVATTO, M.; RIBEIRO, L. P. Manejo das cigarrinhas-das-pastagens com fungos entomopatogênicos. Agropecuária Catarinense, Florianópolis, v. 30, n. 2, p. 42-46, 2017.

MAINA, U. M.; GALADIMA, I. B.; GAMBO, F. M.; ZAKARIA, D. A review on the use of entomopathogenic fungi in the management of insect pests of field crops. Journal of Entomology and Zoology Studies, [S.I.], v. 6, n. 1, p. 27-32, 2018.

MORAES, R. C. B.; HADDAD, M. L.; SILVEIRA NETO, S.; REYES, A. E. L. Software para análise faunística - ANAFAU. In: SIMPÓSIO DE CONTROLE BIOLÓGICO, 8., 2003, São Pedro. Anais [...] Piracicaba: ESALQ/USP, 2003. $195 p$.
MUNSHI, N. A.; HUSSAIN, B.; MALIK, G. N.; YOSUF, M.; FATIMA, N. Efficacy of entomopathogenic fungus Fusarium pallidoroseum (Cooke) Sacc. against gypsy moth (Lymantria obfuscata walker). Journal of Entomology, [S.I.], v. 5, n. 1, p. 59-61, 2008.

PALADINI, A.; TAKIYA, D. M.; URBAN, J. M.; CRYAN, J. R. New world spittlebugs (Hemiptera: Cercopidae: Ischnorhininae): dated molecular phylogeny, classification, and evolution of aposematic coloration. Molecular Phylogenetics and Evolution, [S.I.], v. 120, p. 321-334, 2018.

PERON, A. J.; EVANGELISTA, A. R. Degradação de pastagens em regiões de cerrado. Ciência \& Agrotecnologia, Lavras, v. 28, n. 3, p. 655-661, 2004.

RIBEIRO, L. P.; CASTILHOS, R. V. Manejo de pragas em pastagens: ênfase em pragaschave das gramíneas perenes de verão. Florianópolis: Epagri/DEMC, 2018. 56p. (Boletim técnico, 185).

SILVEIRA NETO, S.; NAKANO, O.; BARBIN, D.; VILLA NOVA, N. A. Manual de ecologia dos insetos. São Paulo: Ceres, 1976. 419p.

THAKUR, A.; SINGH, V.; KAUR, A.; KAUR, S. Suppression of cellular immune response in Spodoptera litura (Lepidoptera: Noctuidae) larvae by endophytic fungi Nigrospora oryzae and Cladosporium uredinicola. Annals of the Entomological Society of America, [S.I.], v. 107, n. 3, p. 674-679, 2014.

VALÉRIO, J. R.; NAKANO, O. Danos causados pelo adulto da cigarrinha Zulia entreriana na produção e qualidade de Brachiaria decumbens. Pesquisa Agropecuária Brasileira, Brasília, DF, v. 23, n. 5, p. 447-453, 1988.

VALÉRIO, J. R.; NAKANO, O. Danos causados pelo adulto da cigarrinha-das-pastagens Zulia entreriana (Berg., 1879) (Homoptera: Cercopidae) em plantas de Brachiaria decumbens Stapf., mantidas em diferentes níveis de umidade. Anais da Sociedade Entomológica do Brasil, Itabuna, v. 16, n. 2, p. 341-350, 1987.

VALÉRIO, J. R.; SOUZA, O. C.; VIEIRA, J. M.; CORRÊA, E. S. Diagnóstico de morte de pastagens nas regiões central e norte do Estado de Mato Grosso. Campo Grande: EMBRAPACNPGC, 2000. 10 p. (Embrapa-CNPGC. Documentos, 98). 